\title{
CORRECTION
}

Check for updates

Cite this: Mater. Chem. Front., 2021, 5, 8398

DOI: 10.1039/d1qm90097h

rsc.li/frontiers-materials

\section{Correction: Novel enzyme-functionalized covalent organic frameworks for the colorimetric sensing of glucose in body fluids and drinks}

\author{
Jie-Yu Yue, ${ }^{\star a}$ Xiu-Li Ding, ${ }^{a}$ Ling Wang, ${ }^{a}$ Run Yang, ${ }^{a}$ Jing-Shu $B i^{a}{ }^{a}$ Ya-Wei Song, ${ }^{b}$ \\ Peng Yang, ${ }^{a}$ Yu Ma*a and Bo Tang ${ }^{\star a}$
}

Correction for 'Novel enzyme-functionalized covalent organic frameworks for the colorimetric sensing of glucose in body fluids and drinks' by Jie-Yu Yue et al., Mater. Chem. Front., 2021, 5, 3859-3866, DOI: 10.1039/D1QM00314C.

The authors regret that the equation for calculating the Michaelis-Menten constant $\left(K_{\mathrm{m}}\right)$ and maximum reaction rate $\left(V_{\max }\right)$ was incorrect. The correct equation is as follows:

$$
\frac{1}{V}=\frac{K_{\mathrm{m}}}{V_{\max }} \frac{1}{C}+\frac{1}{V_{\max }}
$$

where $V$ is the initial reaction rate and $C$ is the concentration of glucose.

The Royal Society of Chemistry apologises for these errors and any consequent inconvenience to authors and readers.

${ }^{a}$ College of Chemistry, Chemical Engineering and Materials Science, Collaborative Innovation Center of Functionalized Probes for Chemical Imaging in Universities of Shandong, Key Laboratory of Molecular and Nano Probes, Ministry of Education, Institutes of Biomedical Sciences, Shandong Normal University, Jinan, 250014, P. R. China. E-mail: tangb@sdnu.edu.cn, may@sdnu.edu.cn, yuejieyu@sdnu.edu.cn

${ }^{b}$ The Affiliated Hospital of Shandong University of Traditional Chinese Medicine, Jinan, Shandong 250014, P. R. China 